\title{
DYNAMICS OF REAL ASTEROID AT THE HECUBA GAP
}

\author{
F. ROIG and S. FERRAZ-MELLO \\ Instituto Astronômico e Geofisico, Universidade de São Paulo \\ Av. Miguel Estéfano 4200, São Paulo, 04301, SP, Brazil
}

Abstract. We study the dynamics of real asteroids at the $2 / 1$ resonance with Jupiter, and find that Griqua-like objects have a short life-time inside the resonance, while Zhongguo group can survive for many $10^{8} \mathrm{yr}$.

The depletion of the Hecuba gap has been recently explained by the existence of a global stochasticity in the region with significant diffusion rates (Nesvorny \& Ferraz-Mello, 1997). This stochasticity is generated by several mechanisms, like the presence of low-order secular resonances (Morbidelli \& Moons, 1993), the overlap of secondary resonances (Henrard et al., 1995), and the overlap of high-order secular resonances with the forced oscillations of the Great-Inequality (Ferraz-Mello et al., 1998). However, the gap is not completely depleted and a few asteroids are found today to be at $2 / 1$ resonance with Jupiter.

In this work, we study the dynamical behaviour of these real asteroids by numerical integration of the equations of motion. The integration scheme was based on the mixed variable symplectic subroutines SWIFT_MVS and SWIFT_RMVS (Levison \& Duncan, 1994). We analyze 15 real asteroids with well-known orbits having a librating critical argument (Table I). Initial conditions for the date 02 21-97 were taken from Bowell's asteroids database and from JPL ephemerides DE403. The simulated Solar System models included perturbations from Venus to Neptune.

The studied objects can be divided in three major groups. Group I contains those asteroids that we call "Griqua-like" objects. They are located at high-eccentricities $(e>0.25)$, have high amplitude of libration $\left(>90^{\circ}\right)$, and normally also high inclination $\left(I>15^{\circ}\right)$. These objects were integrated backwards and forwards for $10^{7} \mathrm{yr}$. Their evolution was characterized by a chaotic variation of the semimajor axis, eccentricity and inclination. The asteroids had several close encounters, within one Hill's sphere, with the inner planets and also with Jupiter, but they did not abandon the resonance ( $a$ stayed librating around the resonant value) until a strong planetary encounter, within 1/10 of Hill's sphere, occurred. After such an encounter (generally with Jupiter), the asteroid was either driven to hit the Sun or to escape from the Solar System. The life-time of these objects inside the resonance is a few $10^{6} \mathrm{yr}$ or less. The only exception is 1362 Griqua, that can survive inside the resonance for more than $5 \times 10^{7} \mathrm{yr}$.

Group II contains the "Zhongguo group". These objects are located at small eccentricities $(e<0.25)$, have low inclinations $\left(I<5^{\circ}\right)$ and moderate amplitudes of libration. Our simulations showed that their behaviour is rather regular and they are able to survive inside the resonance for at least $4 \times 10^{8} \mathrm{yr}$ (or even $10^{9} \mathrm{yr}$ as found by Morbidelli,1996). Finally, Group III contains objects that have a low 
TABLE I

Real asteroids at the Hecuba gap

\begin{tabular}{|c|c|c|c|c|c|}
\hline & I & & II & & III \\
\hline 1362 & Griqua & 3789 & Zhongguo & 4177 & $1987 \mathrm{SS} 1$ \\
\hline 1921 & Pala & & 1975 SX & & 1981 EX11 \\
\hline 1922 & Zulu & & 1990 TH7 & & \\
\hline 3688 & Navajo & & 1993 SK3 & & \\
\hline 5370 & Taranis & & 1994 UD1 & & \\
\hline & 1977 OX & & & & \\
\hline & 1992 АВ & & & & \\
\hline & 1995 QN3 & & & & \\
\hline
\end{tabular}

eccentricity but high inclination. Their life-time is greater than Group I but smaller than Group II, and they are not able to survive inside the resonance for more than $10^{8} \mathrm{yr}$.

Due to their short life-times it is clear that Griqua-like asteroids were injected in the resonance at some time. This process should be very recent and probably not related to the formation of asteroidal families, which are older. It is more probable that Griqua-like asteroids came from the neighbourhood of the resonance, in which case it is likely that a continuous process of low-rate injection of objects into the resonance may exist. This hypothesis is now under study.

\section{References}

Ferraz-Mello S., Michtchenko T.A. and Roig F.: 1998, Astron. J., 116, 1491-1500

Henrard J., Watanabe N. and Moons M.: 1995, Icarus, 115, 336-346

Levison H. and Duncan M.: 1994, Icarus, 108, 18-36

Morbidelli A.: 1996, Astron.J., 111, 2453-2461

Morbidelli A. and Moons M.: 1993, Icarus, 102, 316-332

Nesvorný D. and Ferraz-Mello S.: 1997, A\& A, 320, 672-680 